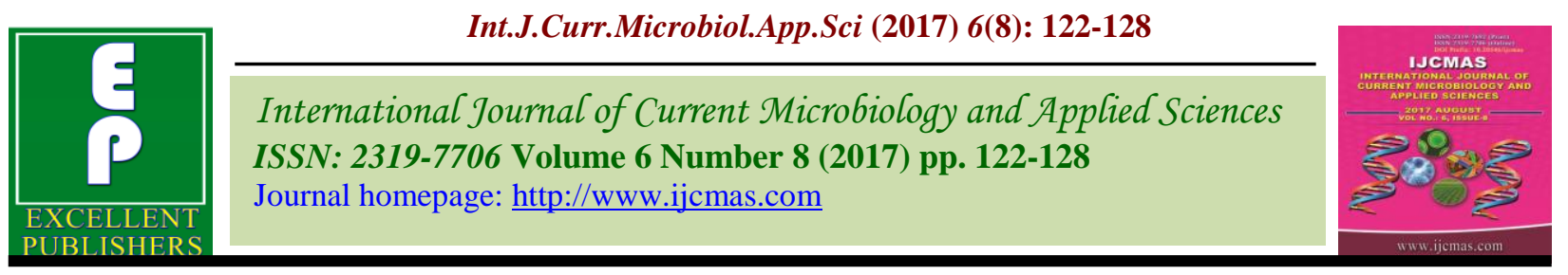

Original Research Article

https://doi.org/10.20546/ijcmas.2017.608.016

\title{
Field Efficacy of Selected Insecticides and Neem Products against Shoot and Fruit Borer [Earias vittella (Fabricius)] on Okra [Abelmoschus esculentus (L.) Moench]
}

\author{
K.A. Rakshith and Ashwani Kumar* \\ Department of Entomology, SHUATS, Allahabad - 211007, India \\ *Corresponding author
}

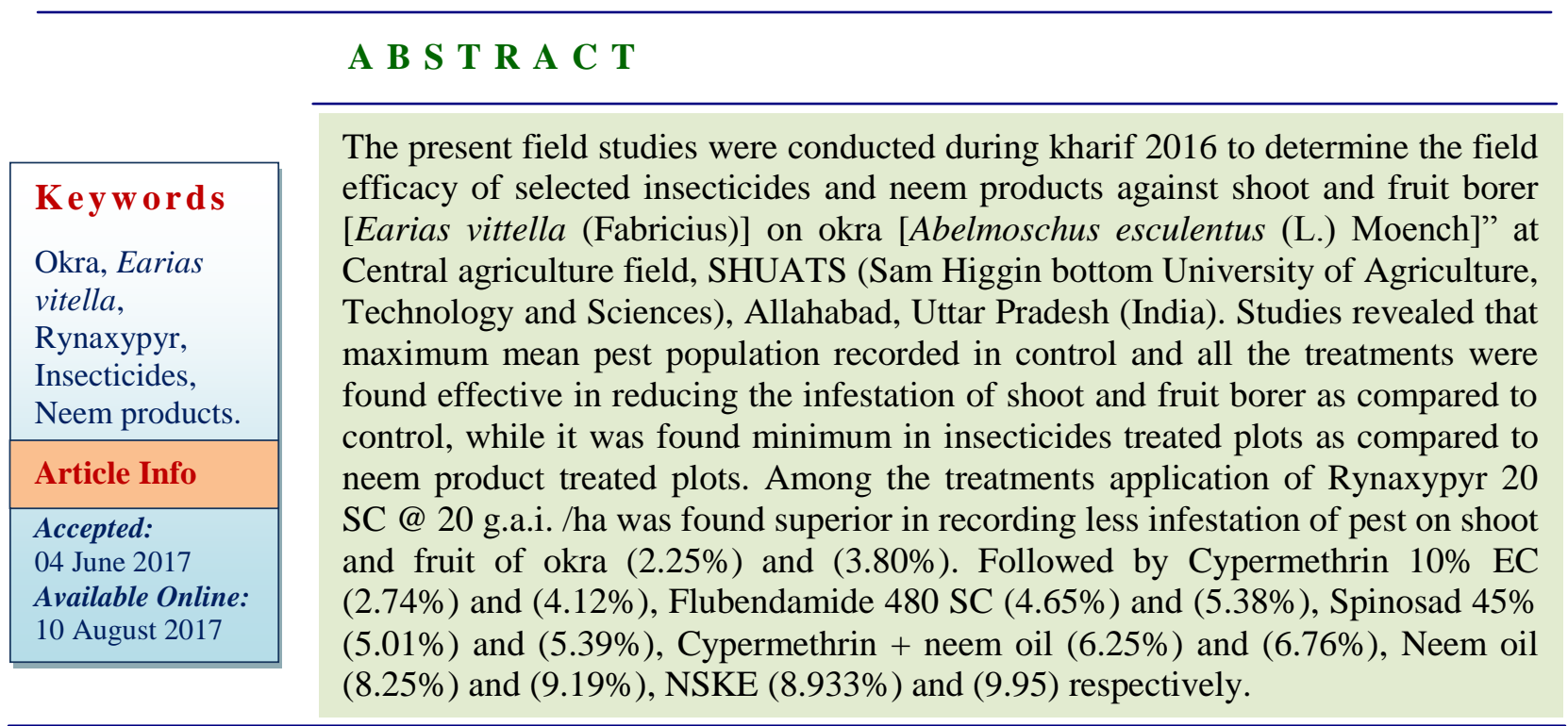

\section{Introduction}

Okra (Abelmoschus esculentus L.) belongs to family Malvaceae or Mallow, which is locally known as Bhendi and Lady's finger worldwide. It is very popular summer vegetable for home gardening while it is also grown commercially throughout the world especially in Indo-Pakistan sub-continent. It is probably originated in Ethiopian region of Africa (Akbar and Khan 2015).It is quite popular in India because of easy cultivation, dependable yield and adaptability to varying moisture conditions. It is an important vegetable crop due to its nutritional, industrial and medicinal value. One of the important limiting factors in the cultivation of okra is insect pests. Okra is susceptible to the attack of various insects from seedling to fruiting stage as high as 72 species of insects have been recorded on okra (Srinivasa and Rajendran, 2003) of which, okra shoot and fruit borer Earias vittella, okra jassid, cut worm, white fly, aphids etc. causes significant damage to the crop. Among these okra shoot and fruit borer (OSFB), Earias vittella is the most serious pest which cause direct damage to tender shoots and fruits. It is reported that about $69 \%$ losses in marketable yield due to attack of this insect pest (Rawat and Sahu, 1973; Radake and Undirwade, 1981). The damage due to shoot and fruit borer, $E$. 
vittella, accounted for about 25 per cent in Uttar Pradesh (Verma et al., 1984) 45 per cent in Karnataka (Srinivasan and Krishna Kumar, 1983), 21.7 to 29.2 per cent in Andhra Pradesh (Neeraja et al., 2004), 22.56 to 22.6 per cent in Tamil Nadu (Praveen and Dhandapani, 2001) and 25.93 per cent in Madhya Pradesh (Dhamdhere et al., 1984). In Rajasthan the yield loss due to borer was estimated as high as 63.53 per cent (Chaudhary and Dadheech, 1989).

In modern days of agriculture, intensification of the vegetable cultivation has created tremendous pest problems; this has led to high pesticide use, often with no reduction in pests attack, making vegetable production more and more dependent on pesticides. Conventionally farmers are using various types of synthetic chemical insecticides to control okra shoot and fruit borer. But due to the unconscious and unjustified use of synthetic pesticides create several problems in agro-ecosystem such as direct toxicity to beneficial insects, fishes, and man (Goodland et al., 1985). The intensive use of highly toxic and broad spectrum pesticides and their repeated use alone have resulted in the development of resistance in the insect pest, and disturbance to the agroecosystem by affecting the nontargets (Dittrich et al., 1990). Elimination of natural enemies, environmental disharmony, consequently it becomes more difficult to manage key pests and secondary pests. Frequent pickings, high operational cost and indiscriminate use of insecticides resulted in the presence of residues in these vegetables (Arora, 2009) are limiting factors for the chemical control of pests of okra. Due to the presence of pesticide residues in the final commodity, there is a risk of rejection of whole consignments during export. This is manifested in a strong demand for reduced use of pesticides from general public, government and in an increasing number of countries. To overcome these problems, use of resistant or tolerant variety and use of bio pesticide, botanicals are safe to mammals, safe to natural enemies, which fit well in the IPM concept, is the need of the hour. There is also an increasing demand for healthy food which boosts organic production. So, any non-chemical strategy for managing fruit borer could be a good approach. And chemical pesticides are usually the key tool for combating pests, also it is now urgently need to use safe and effective biodegradable pesticides with less toxic effects on non-target organisms.

Botanical pesticides possess an array of properties including insecticidal activity and insect growth regulatory activity against many insect and mite pests (Rajasekaran and Kumaraswami, 1985; Prakash and Rao, 1989). The neem products with half the dose of conventional insecticides has resulted in more efficient control than insecticides alone (Sinzogan et al., 2006). Neem oil produced nontoxic effects after spray and acted as antifeedent, growth inhibitor and oviposition deterrent against insect's pests of okra and cotton (Ahmed et al., 1995). Low mammalian toxicity, no reported development of resistance, less hazardous to non-target organisms, no pest resurgence problem, no adverse effect on plant growth, negligible application risks, low cost and easy availability are the advantages of plant products over synthetic chemicals.

Indiscriminate use of several insecticides in agricultural field creates problem in the natural ecosystem, environmental pollution, pest resistance and health hazards etc. Due to this, only selective chemicals used in order to avoid indiscriminate use of pesticides. Also a number of previous studies on the sustainable management of insect pests on okra ecosystem through IPM technologies based on the intensive use of bio pesticides and other environmentally safer botanicals 
considerable success in mitigating the insect pests damage, reduction in the pesticide usage and restoration of ecological balance (Sardana et al., 2005; Preetha and Nadarajan, 2007; Reddy, 2013) keeping in view, the above, a study entitled "Field efficacy of selected insecticides and neem products againstshoot and fruit borer [Earias vittella (Fabricius)] major insect pests of okra [Abelmoschus esculentus (L.) Moench]" is taken.

\section{Materials and Methods}

Studies on the "Field efficacy of selected insecticides and neem products against shoot and fruit borer, (Earias vittellaFabricius) on okra [Abelmoschus esculentus (L.) Moench]'. In Allahabad region under field condition was carried out at the Central field, SHUATS (Sam Higgin bottom University of Agriculture, Technology and Sciences), Allahabad, Uttar Pradesh, India. Experiment was, Eight treatments consisting of T1Neemoil 5\%, T2-Flubendamide 48 SC, T3Cypermethrin 25EC, T4-NSKE 5\%, T5Spinosad,T6-Cypermethrin+neemoil, T7Rynaxypyr 20 SC, T8-control were tested to compare the efficacy of selected insecticides and neem products against shoot and fruit borer [Earias vittella (Fabricius)] on okra [Abelmoschus esculentus (L.) Moench]. The okra variety BND 777 was sown @ 10 kg.ha ${ }^{-1}$ by dibbling method with spacing of $45 \mathrm{~cm}$ between row to row and $30 \mathrm{~cm}$ between plant to plant by placing 2-3 seeds per hill at depth of $4 \mathrm{~cm}$.

\section{Shoot and fruit borer}

(Field Efficacy of treatment) the incidence of the borer on the shoot and the fruit was recorded from the five randomly selected plants. Observations were recorded one day before spray and $3^{\text {rd }}, 7^{\text {th }}, 11^{\text {th }}$, days after spraying. The extent of the damage, computed by using the formula;
Number of damaged shoots

Percent shoot infestation - --------------- X 100

Total number of shoots

Number of damaged fruits

Percent fruit infestation - ---------------- X 100

Total number of fruits

\section{Results and Discussion}

\section{Percent shoot infestation (Assessment of infestation: Number basis)}

The data on the percent infestation of shoot borer of $\mathrm{I}^{\mathrm{st}}$ and $\mathrm{II}^{\mathrm{nd}}$ spray pooled mean revealed that all the chemical treatments were significantly superior over control. Among all the treatments lowest percent infestation of shoot and fruit borer was recorded in Rynaxypyr (2.25\%) and Cypermethrin $(2.74 \%)$ are at par with each other, followed by Flubendamide (4.65\%) and Spinosad $(5.01 \%)$ are at par with each other, Cypermethrin + Neemoil $(6.25 \%)$, followed by Neemoil $(8.25 \%)$ and NSKE $(8.93 \%)$ is the least effective among all the treatments and are statistically at par with each other.

Percent fruit infestation (Assessment of infestation: Number basis)

The efficacy of certain chemical insecticides and neem products against percent fruit infestation of shoot and fruit borer are depicted in table. The data on the percent infestation of fruit borer on IInd and IIIrd spray pooled mean revealed that all the chemical treatments were significantly superior over control. Among all the treatments lowest percent infestation of shoot and fruit borer was recorded in Rynaxypyr (3.80\%) and Cypermethrin (4.12\%) are at par with each other, followed by Flubendamide $(5.38 \%)$ and Spinosad $(5.39 \%)$ are at par with each other, Cypermethrin+ Neemoil (6.76\%), followed by Neemoil $(9.19 \%)$ and NSKE $(9.95 \%)$ is the least effective among all the treatments. 


\begin{tabular}{|c|c|c|c|c|c|c|c|c|c|c|c|c|c|}
\hline \multirow{2}{*}{\multicolumn{2}{|c|}{ Treatments }} & \multirow{2}{*}{$\begin{array}{c}\text { Concentration/ } \\
\text { Dose }\end{array}$} & \multicolumn{5}{|c|}{$\mathrm{I}^{\mathrm{st}}$ Spray(\% infestation of shoots) } & \multicolumn{5}{|c|}{ II $^{\text {nd }}$ Spray (\% infestation of shoots) } & \multirow{2}{*}{$\begin{array}{c}\text { Over all } \\
\text { mean }\end{array}$} \\
\hline & & & $1 \mathrm{DBS}$ & 3 DAS & 7 DAS & 11 DAS & Mean & $1 \mathrm{DBS}$ & 3 DAS & 7 DAS & 11DAS & Mean & \\
\hline $\mathrm{T} 1$ & Neemoil & $3 \mathrm{ml} / \mathrm{L}$ & $\begin{array}{c}9.67 \\
(18.12)\end{array}$ & $\begin{array}{c}6.87 \\
(15.19)\end{array}$ & $\begin{array}{c}7.14 \\
(15.50)\end{array}$ & $\begin{array}{c}7.69 \\
(16.10)\end{array}$ & $\begin{array}{c}7.25 \\
(15.631)\end{array}$ & $\begin{array}{c}11.32 \\
(19.66)\end{array}$ & $\begin{array}{c}8.33 \\
(16.77)\end{array}$ & $\begin{array}{c}8.80 \\
(17.26)\end{array}$ & $\begin{array}{c}8.97 \\
(17.43)\end{array}$ & $\begin{array}{c}8.714 \\
(17.169)\end{array}$ & $\begin{array}{c}8.25 \\
(16.69)\end{array}$ \\
\hline $\mathrm{T} 2$ & $\begin{array}{c}\text { Flubendamide } \\
48 \mathrm{SC}\end{array}$ & $4 \mathrm{ml} / \mathrm{L}$ & $\begin{array}{c}9.23 \\
(17.68)\end{array}$ & $\begin{array}{c}3.47 \\
(11.77)\end{array}$ & $\begin{array}{c}3.89 \\
(11.38)\end{array}$ & $\begin{array}{c}5.96 \\
(14.13)\end{array}$ & $\begin{array}{c}4.67 \\
(12.489)\end{array}$ & $\begin{array}{c}8.15 \\
(16.58)\end{array}$ & $\begin{array}{c}3.37 \\
(10.57)\end{array}$ & $\begin{array}{c}4.81 \\
(12.67)\end{array}$ & $\begin{array}{c}5.96 \\
(14.13)\end{array}$ & $\begin{array}{c}4.651 \\
(12.454)\end{array}$ & $\begin{array}{c}4.65 \\
(12.46)\end{array}$ \\
\hline T3 & $\begin{array}{c}\text { Cypermethrin } \\
\text { 25EC }\end{array}$ & $2 \mathrm{ml} / \mathrm{L}$ & $\begin{array}{c}8.39 \\
(16.83)\end{array}$ & $\begin{array}{c}2.79 \\
(8.73)\end{array}$ & $\begin{array}{c}2.64 \\
(8.87)\end{array}$ & $\begin{array}{c}3.46 \\
(10.73)\end{array}$ & $\begin{array}{c}2.79 \\
(9.627)\end{array}$ & $\begin{array}{c}6.28 \\
(14.51)\end{array}$ & $\begin{array}{c}2.11 \\
(8.35)\end{array}$ & $\begin{array}{c}2.36 \\
(8.84)\end{array}$ & $\begin{array}{c}3.46 \\
(10.73)\end{array}$ & $\begin{array}{c}2.714 \\
(9.483)\end{array}$ & $\begin{array}{c}2.74 \\
(9.53)\end{array}$ \\
\hline $\mathrm{T} 4$ & NSKE & $5 \mathrm{~g} / \mathrm{L}$ & $\begin{array}{c}9.02 \\
(17.48)\end{array}$ & $\begin{array}{c}7.09 \\
(15.44)\end{array}$ & $\begin{array}{c}7.69 \\
(16.10)\end{array}$ & $\begin{array}{c}8.39 \\
(16.83)\end{array}$ & $\begin{array}{c}7.72 \\
(16.139)\end{array}$ & $\begin{array}{c}12.20 \\
(20.29)\end{array}$ & $\begin{array}{c}9.49 \\
(17.94)\end{array}$ & $\begin{array}{c}9.43 \\
(17.88)\end{array}$ & $\begin{array}{c}9.55 \\
(18.00)\end{array}$ & $\begin{array}{c}9.493 \\
(17.945)\end{array}$ & $\begin{array}{c}8.93 \\
(17.39)\end{array}$ \\
\hline T5 & Spinosad & $0.5 \mathrm{~g} / \mathrm{L}$ & $\begin{array}{c}9.48 \\
(17.94)\end{array}$ & $\begin{array}{c}4.31 \\
(11.99)\end{array}$ & $\begin{array}{c}4.41 \\
(12.12)\end{array}$ & $\begin{array}{c}5.66 \\
(13.76)\end{array}$ & $\begin{array}{c}4.83 \\
(12.707)\end{array}$ & $\begin{array}{c}7.69 \\
(16.10)\end{array}$ & $\begin{array}{c}4.19 \\
(11.81)\end{array}$ & $\begin{array}{c}5.32 \\
(13.34)\end{array}$ & $\begin{array}{c}5.66 \\
(13.76)\end{array}$ & $\begin{array}{c}5.095 \\
(13.045)\end{array}$ & $\begin{array}{c}5.01 \\
(12.94)\end{array}$ \\
\hline T6 & $\begin{array}{c}\text { Cypermethrin+ } \\
\text { neem oil }\end{array}$ & $1+1.5 \mathrm{ml} / \mathrm{L}$ & $\begin{array}{c}9.16 \\
(17.61)\end{array}$ & $\begin{array}{c}5.10 \\
(13.06)\end{array}$ & $\begin{array}{c}5.55 \\
(12.81)\end{array}$ & $\begin{array}{c}6.15 \\
(14.36)\end{array}$ & $\begin{array}{c}5.506 \\
(13.571)\end{array}$ & $\begin{array}{c}8.33 \\
(16.77)\end{array}$ & $\begin{array}{c}4.68 \\
(12.50)\end{array}$ & $\begin{array}{c}6.89 \\
(15.22)\end{array}$ & $\begin{array}{c}7.69 \\
(16.10)\end{array}$ & $\begin{array}{c}6.623 \\
(14.914)\end{array}$ & $\begin{array}{c}6.25 \\
(14.48)\end{array}$ \\
\hline $\mathrm{T} 7$ & $\begin{array}{c}\text { Rynaxypyr } \\
20 \text { SC }\end{array}$ & $4 \mathrm{ml} / \mathrm{L}$ & $\begin{array}{c}9.55 \\
(18.00)\end{array}$ & $\begin{array}{c}2.20 \\
(8.54)\end{array}$ & $\begin{array}{c}2.02 \\
(8.18)\end{array}$ & $\begin{array}{c}2.53 \\
(9.15)\end{array}$ & $\begin{array}{c}2.26 \\
(8.650)\end{array}$ & $\begin{array}{c}6.21 \\
(14.43)\end{array}$ & $\begin{array}{r}1.92 \\
(7.97)\end{array}$ & $\begin{array}{c}2.23 \\
(8.60)\end{array}$ & $\begin{array}{c}2.58 \\
(9.24)\end{array}$ & $\begin{array}{c}2.247 \\
(8.621)\end{array}$ & $\begin{array}{c}2.25 \\
(8.63)\end{array}$ \\
\hline $\mathrm{T} 8$ & Untreated & - & $\begin{array}{c}9.16 \\
(17.61)\end{array}$ & $\begin{array}{c}10.21 \\
(18.64)\end{array}$ & $\begin{array}{c}11.03 \\
(19.40)\end{array}$ & $\begin{array}{c}12.13 \\
(20.38)\end{array}$ & $\begin{array}{c}11.208 \\
(19.560)\end{array}$ & $\begin{array}{c}14.89 \\
(22.70)\end{array}$ & $\begin{array}{c}15.49 \\
(23.17)\end{array}$ & $\begin{array}{c}16.43 \\
(23.91)\end{array}$ & $\begin{array}{c}16.76 \\
(24.16)\end{array}$ & $\begin{array}{c}16.268 \\
(23.787)\end{array}$ & $\begin{array}{r}14.59 \\
(22.46)\end{array}$ \\
\hline & F- test & & NS & $\mathbf{S}$ & $\mathbf{S}$ & $\mathbf{S}$ & $\mathbf{S}$ & NS & $\mathbf{S}$ & $\mathbf{S}$ & $\mathbf{S}$ & $\mathbf{S}$ & $\mathbf{S}$ \\
\hline & S. Ed. ( $( \pm)$ & & 0.37 & 0.52 & 0.38 & 0.28 & 0.17 & 1.05 & 0.26 & 0.34 & 0.40 & 0.26 & 0.39 \\
\hline & C. D. $(P=0.05)$ & & 1.13 & 1.40 & 0.66 & 0.78 & 0.52 & 2.72 & 0.79 & 1.03 & 1.20 & 0.72 & 0.75 \\
\hline & $\begin{array}{l}\text { in parenthesis are } \\
\text {-Days before spr }\end{array}$ & transformed & . & 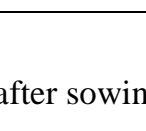 & & & & & & & & & \\
\hline
\end{tabular}




\begin{tabular}{|c|c|c|c|c|c|c|c|c|c|c|c|c|c|}
\hline \multirow{2}{*}{\multicolumn{2}{|c|}{ Treatments }} & \multirow{3}{*}{$\begin{array}{c}\text { Concentration/ } \\
\text { Dose }\end{array}$} & \multicolumn{5}{|c|}{$\mathrm{II}^{\mathrm{nd}}$ Spray (\% infestation of fruits) } & \multicolumn{5}{|c|}{ III $^{\text {rd }}$ Spray(\% infestation of fruits) } & \multirow{3}{*}{$\begin{array}{c}\begin{array}{l}\text { Over all } \\
\text { mean }\end{array} \\
9.19 \\
(17.65)\end{array}$} \\
\hline & & & \multirow{2}{*}{$\begin{array}{c}1 \text { DBS } \\
10.34 \\
(18.76)\end{array}$} & \multirow{2}{*}{$\begin{array}{c}\text { 3 DAS } \\
7.59 \\
(15.99)\end{array}$} & \multirow{2}{*}{$\begin{array}{c}7 \text { DAS } \\
7.69 \\
(16.10)\end{array}$} & \multirow{2}{*}{$\begin{array}{c}11 \text { DAS } \\
7.84 \\
(16.31)\end{array}$} & \multirow{2}{*}{$\begin{array}{c}\text { Mean } \\
7.731 \\
(16.14)\end{array}$} & \multirow{2}{*}{$\begin{array}{c}\text { 1 DBS } \\
17.04 \\
(24.38)\end{array}$} & \multirow{2}{*}{$\begin{array}{c}\text { 3 DAS } \\
11.39 \\
(19.72)\end{array}$} & \multirow{2}{*}{$\begin{array}{r}7 \text { DAS } \\
11.66 \\
(19.97)\end{array}$} & \multirow{2}{*}{$\begin{array}{c}\text { 11DAS } \\
12.19 \\
(20.43)\end{array}$} & \multirow{2}{*}{$\begin{array}{c}\text { Mean } \\
11.76 \\
(20.05)\end{array}$} & \\
\hline $\mathrm{T} 1$ & Neemoil & & & & & & & & & & & & \\
\hline $\mathrm{T} 2$ & $\begin{array}{c}\text { Flubendamide } \\
48 \mathrm{SC}\end{array}$ & $4 \mathrm{ml} / \mathrm{L}$ & $\begin{array}{c}9.67 \\
(18.12)\end{array}$ & $\begin{array}{c}4.76 \\
(12.60)\end{array}$ & $\begin{array}{c}5.26 \\
(13.26)\end{array}$ & $\begin{array}{c}5.47 \\
(13.53)\end{array}$ & $\begin{array}{c}5.181 \\
(13.15)\end{array}$ & $\begin{array}{c}13.79 \\
(21.80)\end{array}$ & $\begin{array}{c}5.35 \\
(13.38)\end{array}$ & $\begin{array}{c}5.55 \\
(13.63)\end{array}$ & $\begin{array}{c}6.55 \\
(14.83)\end{array}$ & $\begin{array}{c}5.84 \\
(13.99)\end{array}$ & $\begin{array}{c}5.38 \\
(13.41)\end{array}$ \\
\hline $\mathrm{T} 4$ & NSKE & $5 \mathrm{~g} / \mathrm{L}$ & $\begin{array}{c}10.71 \\
(19.10)\end{array}$ & $\begin{array}{c}8.45 \\
(16.89)\end{array}$ & $\begin{array}{c}8.69 \\
(17.15)\end{array}$ & $\begin{array}{c}8.82 \\
(17.28)\end{array}$ & $\begin{array}{c}8.653 \\
(17.10)\end{array}$ & $\begin{array}{c}18.51 \\
(25.48)\end{array}$ & $\begin{array}{c}12.5 \\
(20.70)\end{array}$ & $\begin{array}{c}12.32 \\
(20.55)\end{array}$ & $\begin{array}{c}12.90 \\
(21.05)\end{array}$ & $\begin{array}{c}12.56 \\
(20.75)\end{array}$ & $\begin{array}{c}9.95 \\
(18.38)\end{array}$ \\
\hline $\mathrm{T} 5$ & Spinosad & $0.5 \mathrm{~g} / \mathrm{L}$ & $\begin{array}{c}9.23 \\
(17.68)\end{array}$ & $\begin{array}{c}4.83 \\
(12.70)\end{array}$ & $\begin{array}{c}5.17 \\
(13.14)\end{array}$ & $\begin{array}{c}5.45 \\
(13.50)\end{array}$ & $\begin{array}{c}5.142 \\
(13.10)\end{array}$ & $\begin{array}{c}13.95 \\
(21.93)\end{array}$ & $\begin{array}{c}5.55 \\
(13.63)\end{array}$ & $\begin{array}{c}5.45 \\
(13.50)\end{array}$ & $\begin{array}{c}6.32 \\
(14.57)\end{array}$ & $\begin{array}{c}5.85 \\
(13.99)\end{array}$ & $\begin{array}{c}5.39 \\
(13.42)\end{array}$ \\
\hline T6 & $\begin{array}{c}\text { Cypermethrin+ne } \\
\text { em oil }\end{array}$ & $1+1.5 \mathrm{ml} / \mathrm{L}$ & $\begin{array}{c}9.37 \\
(17.83)\end{array}$ & $\begin{array}{c}5.66 \\
(13.76)\end{array}$ & $\begin{array}{c}6.64 \\
(14.96)\end{array}$ & $\begin{array}{c}6.97 \\
(15.31)\end{array}$ & $\begin{array}{c}6.521 \\
(14.79)\end{array}$ & $\begin{array}{c}14.63 \\
(22.49)\end{array}$ & $\begin{array}{c}6.66 \\
(14.96)\end{array}$ & $\begin{array}{c}6.81 \\
(15.13)\end{array}$ & $\begin{array}{c}7.77 \\
(16.19)\end{array}$ & $\begin{array}{c}7.26 \\
(15.63)\end{array}$ & $\begin{array}{c}6.76 \\
(15.07)\end{array}$ \\
\hline $\mathrm{T} 7$ & $\begin{array}{c}\text { Rynaxypyr } \\
20 \text { SC }\end{array}$ & $4 \mathrm{ml} / \mathrm{L}$ & $\begin{array}{c}8.21 \\
(16.66)\end{array}$ & $\begin{array}{c}3.43 \\
(10.76)\end{array}$ & $\begin{array}{c}3.52 \\
(10.82)\end{array}$ & $\begin{array}{c}3.70 \\
(11.09)\end{array}$ & $\begin{array}{c}3.571 \\
(10.89)\end{array}$ & $\begin{array}{c}11.25 \\
(19.59)\end{array}$ & $\begin{array}{c}4.16 \\
(11.77)\end{array}$ & $\begin{array}{c}4.28 \\
(11.94)\end{array}$ & $\begin{array}{c}4.76 \\
(12.60)\end{array}$ & $\begin{array}{c}4.39 \\
(12.09)\end{array}$ & $\begin{array}{c}3.80 \\
(11.25)\end{array}$ \\
\hline $\mathrm{T} 8$ & Untreated & - & $\begin{array}{c}13.11 \\
(21.23)\end{array}$ & $\begin{array}{c}14.51 \\
(22.39)\end{array}$ & $\begin{array}{c}14.81 \\
(16.10)\end{array}$ & $\begin{array}{c}15.04 \\
(22.63)\end{array}$ & $\begin{array}{l}14.732 \\
(22.57)\end{array}$ & $\begin{array}{c}22.23 \\
(28.12)\end{array}$ & $\begin{array}{c}25.86 \\
(30.56)\end{array}$ & $\begin{array}{c}27.27 \\
(31.48)\end{array}$ & $\begin{array}{c}27.86 \\
(31.86)\end{array}$ & $\begin{array}{c}27.01 \\
(31.13)\end{array}$ & $\begin{array}{c}18.16 \\
(25.22)\end{array}$ \\
\hline & F- test & & NS & $\mathbf{S}$ & $\mathbf{S}$ & $\mathbf{S}$ & $\mathbf{S}$ & NS & $\mathbf{S}$ & $\mathbf{S}$ & $\mathbf{S}$ & $\mathbf{S}$ & $\mathbf{S}$ \\
\hline & S. Ed. $( \pm)$ & & 0.93 & 0.24 & 0.34 & 0.22 & 0.19 & 0.27 & 0.25 & 0.24 & 0.29 & 0.18 & 0.18 \\
\hline & C. D. $(P=0.05)$ & & 2.81 & 0.72 & 1.02 & 0.68 & 0.59 & 4.62 & 0.75 & 0.74 & 0.89 & 0.55 & 0.56 \\
\hline
\end{tabular}


Similar readings were found with Chowdary et al., (2010) showed among the newer insecticide molecules evaluated, Rynaxypyr $20 \mathrm{SC}$ were superior in recording less larval populations, lower fruit damage (7.80 and $10.51 \%)$.

Singh et al., (2015) recorded Cypermethrin 25 EC recorded the lowest percentage of shoot and fruit damage followed by Spinosad 45 SC. some extent corroborate with the present findings.

Nalini and Kumar (2016) reported against shoot and fruit borer, Earias vittella. Minimum percent of shoot infestation and percent fruit infestation were observed in cypermethrin with $(3.60 \%$ and $6.34 \%)$.

In shoot and fruit borer, all the three sprays revealed that Rynaxypyr and Cypermethrin were at par with each other and was found to be more effective than other treatments.

From the critical analysis of the present findings it can be concluded that selected insecticides like Rynaxypyr and Cypermethrin, Flubendamide and Spinosad can be suitably incorporated in pest management schedule against Earias vitella as an effective tool under chemical control, and combination treatments like Cypermethrin + Neemoil, botanicals like Neemoil and NSKE are also to be incorporated in pest management in order to avoid indiscriminate use of pesticides causing pollution in the environment and not much harmful to beneficial insects and in increasing cost effectiveness. And these synthetic chemicals are better than botanicals in reducing pest population level.

\section{References}

Abhishek kumar Chaudhary, Ashwani kumar, Umesh Chandra and Rahul singh
(2016). Bioefficacy of some indigenous products and spinosad in the management of okra shoot and fruit borer (Earias vitelli Fabricius). ISSN; 0974-4908 Res. Environ. Life Sci. 9(2) 137-139.

Bansode, A.G., Patil, C.S. and Jadhav, S.S. (2015). Efficacy of insecticides against shoot and fruit borer, Earias vittella $F$. infesting okra. Pest Management in Horticultural Ecosystems, Vol. 21, No. 1 pp 106-109.

Challa Nalini and Ashwani Kumar (2016).Population dynamics and comparative efficacy of certain chemicals and bio pesticides against okra shoot and fruit borer shoot and fruit borer (Earias vitella).The bioscan11(3): 1589-1592.

Dabhi, M. V., Acharya, M. F., Koshiya, D. J. and Khanpara, A. V. (2012). Bioefficacy of various insecticides against okra shoot and fruit borer, Earias vittella (Fab.). AGRES- An international-Journal.1 (1): ISSN 22779663.

Garcia, P., (1964), Memoir of the experiments carried out against $E$. insulana and $P$. gossypiella and other pests of cotton, campaign of 1962. Ann. Inst. Nac. Invest Agron, 12: 155-176.

Gonde, A.D., Wargantiwar, R.K., Kumar, A. and Burange, P.S. (2013). Screening varieties of Okra (Abelmescus esculentus (L.)Moench) against Earias vitelli (Fabrcius) in Allahabad (U.P), India. J.Soils and Crops 23(1) 69-72.

Gautam, H.K., Singh1 N.N. and Rai, A.B. (2015). Effect of some plant extract and an insecticide on the incidence of Earias vittella in okra. Indian J. Agric. Res., 49 (2): 175-179.

Katti Pramod and Surpur Shwetha (2015). Field bio efficacy of flubendiamide 480 SC against okra fruit and shoot borer, Earias vitella (Fab.) during Rabi 
season. Internat. J. Plant Protec., 8(2): 319-323.

Pardeshi, A.M., Bharodia, R.K., Joshi, M.D., Makadia, R.R. AND Kate, A.O. (2010).Chemical Control of Earias vittella (Fabricius) on Okra. International Journal of Plant Protection, Vol. 2 No. 2: 231-233.

Padwal, K. G. and Ashwani Kumar (2014). Efficacy of plant products and combinations with cypermethrin in management of Earias vittella of okra. Annals of Plant Protection Sciences, 22(1): 73-75.

Rajesh Chowdary, L., Bhemanna, M. AND Ranjith kumar, L. (2010). Bio efficacy of rynaxypyr (Coragen) $20 \mathrm{SC}$ against fruit borer Helicoverpa armigera (Hubner) in okra. International Journal of Plant Protection (October,), Vol. 3 No. 2: 379-381.ISSN: 0974-0163.

Sabyasachi Pal, Tarak Brambha Maji and Palash Mondal (2013). Incidence of insect pest on okra, Abelmoschus esculentus (L) Moench in red lateritic zone of West Bengal. The Journal of Plant Protection Sciences, 5(1): 59-64.

Tulankar, K.P, Gonde, A.D., Wargantiwar, R.K., Kumar A. and Burange, P.S. (2012). Ecofriendly Approach for the Management of Shoot and Fruit Borer, Earias vittella (Fab.) on Okra in Allahabad (UP) ISSN: 09762485Volume: 5, Issue: 3.

\section{How to cite this article:}

Rakshith, K.A. and Ashwani Kumar. 2017. Field Efficacy of Selected Insecticides and Neem Products against Shoot and Fruit Borer [Earias vittella (Fabricius)] on Okra [Abelmoschus esculentus (L.) Moench]. Int.J.Curr.Microbiol.App.Sci. 6(8): 122-128. doi: https://doi.org/10.20546/ijcmas.2017.608.016 